\title{
Nonlinear stationary waves and solitary waves in fluid systems with long-range forces
}

\author{
Fred C. Adams, Marco Fatuzzo and Richard Watkins \\ Physics Department, University of Michigan, Ann Arbor, MI 48109, USA
}

Received 3 June 1993; accepted for publication 17 September 1993

Communicated by D.D. Holm

\begin{abstract}
We study a class of nonlinear model equations which represent one-dimensional fluid systems with long range forces (such as gravity). We present elementary theorems which constrain the allowed types of stationary wave and solitary wave solutions for this class of systems.
\end{abstract}

\section{Introduction}

A wide variety of nonlinear wave structures can exist in fluid systems where self-gravity is important, but such motions have not been well studied. These waves are most likely to occur in astrophysical settings, although many other physical systems with long range forces can mathematically resemble this problem (see below). In this Letter, we consider a class of nonlinear equations which model self-gravitating fluid systems in one spatial dimension. Our goal is to find general results which show how physical properties of such systems determine the allowed types of stationary wave solutions.

Fluid motions which simultaneously include both self-gravity and nonlinear effects can exhibit interesting behavior. In general, most nonlinear waves in fluids tend to steepen and shock $[1,2]$. On the other hand, self-gravity leads to dispersion [3] which tends to spread out wave packets. In principle, fluids with self-gravity can reach a balance between nonlinear steepening and gravitational dispersion. This balance leads to the possible existence of both nonlinear stationary waves and solitary waves in the fluid. However, previous work in this area [4-6] has shown that the simplest possible system (i.e., eqs. (1) and (2) below coupled with standard one-dimensional gravity) cannot exhibit solitary wave behavior. The physical reason for this lack of solitary waves is that the gravitational force in one spatial dimension does not decrease in the asymptotic limit $x \rightarrow \infty$; on sufficiently large spatial scales, the inward pull of gravity always dominates and such systems are thus doomed to collapse.

In this contribution, we study a general class of model equations which represent self-gravitating fluid systems in one spatial dimension. In particular, we show that many systems can be described by the usual equations of hydrodynamics coupled with a modified form of the Poisson equation for the gravitational potential. One motivation for using a modified Poisson equation is to model two-dimensional gravitational effects (e.g., a finite extent of the wave profile in the transverse direction) while retaining a one-dimensional theory. We present four elementary theorems which constrain the allowed types of wave behavior accessible to these physical systems. These results elucidate the properties that a system must have in order to exhibit stationary waves and solitary waves.

\section{General formulation}

In this section, we introduce a class of model equations for the study of nonlinear waves in self-gravitating fluid systems. We begin with the equations of motion for a fluid. In one spatial dimension, the con- 
tinuity equation and the force equation take the forms

$\frac{\partial \rho}{\partial t}+\frac{\partial}{\partial x}(\rho u)=0$

$\frac{\partial u}{\partial t}+u \frac{\partial u}{\partial x}+\frac{1}{\rho} \frac{\partial p}{\partial x}+\frac{\partial \psi}{\partial x}=0$,

where $\rho$ is the mass density, $u$ is the velocity, $\psi$ is the gravitational potential, and $p$ is the pressure. All quantities have been written in dimensionless form. We take the pressure to have a general barotropic form

$p=p(\rho)$,

which includes most equations of state of interest. To close the system of equations, we must include the Poisson equation for the gravitational potential. In this paper, we consider a class of systems with the Poisson equation written in the generalized form

$\frac{\partial^{2} \psi}{\partial x^{2}}=q(\rho)$

where $q(\rho)$ is a function of the density $\rho$. We denote the quantity $q(\rho)$ as the charge density. We use this terminology because, in general, whatever appears on the right hand side of a Poisson equation is often called "the charge density". Notice that the charge density defined here is not the electric charge density. Notice also that the choice $q=\rho$ gives us back the usual one-dimensional Poisson equation.

Equations (1)-(4) completely determine the system once the equation of state $p(\rho)$ and the form of the charge density $q(\rho)$ are specified. We denote theories of this type as "charge density theories". For most of this paper, we keep the forms for $p(\rho)$ and $q(\rho)$ arbitrary and present general results. In section 3 , however, we show that many different physical systems can be described by charge density theories and we present specific forms of $q(\rho)$ for several examples.

We focus this current discussion on stationary waves, which correspond to traveling waves of permanent form. For these waves, the fluid fields are functions of the quantity

$\xi=x-v_{0} t$,

where $v_{0}$ is the (dimensionless) speed of the wave. Next, we introduce a new velocity variable $v=u-v_{0}$,

which is simply the speed of the fluid relative to the speed $v_{0}$ of the wave. Using these definitions, we integrate the continuity equation (1) to obtain the relation

$\rho v=A$,

where the constant of integration $A$ is the "Mach number" of the wave.

We can reduce the system of equations described above to a single nonlinear differential equation for the density $\rho$. If we differentiate the force equation (2) with respect to $\xi$ and use the generalized Poisson equation (4) to eliminate the potential, we obtain

$$
\begin{aligned}
& \rho \rho_{\xi \xi}\left(\rho^{2} \frac{\partial p}{\partial \rho}-A^{2}\right)+\rho_{\xi}^{2}\left(3 A^{2}-\rho^{2} \frac{\partial p}{\partial \rho}+\rho^{3} \frac{\partial^{2} p}{\partial \rho^{2}}\right) \\
& +\rho^{4} q(\rho)=0,
\end{aligned}
$$

where we have eliminated the velocity dependence by using the solution (7) to the continuity equation (and where differentiation is now denoted by subscripts). The equation of motion (8) is the fundamental equation governing stationary waves in charge density theories. Fortunately, this highly nonlinear equation can be integrated to obtain

$\frac{1}{2} \rho_{\xi}^{2}=\rho^{6}\left(\rho^{2} \frac{\partial p}{\partial \rho}-A^{2}\right)^{-2} f(\rho) \equiv \mathscr{F}(\rho)$,

where we have defined $f(\rho)$ to be an integral that depends on the form of the charge density $q(\rho)$, i.e.,

$f(\rho)=\int^{\rho} \mathrm{d} \rho \frac{q(\rho)}{\rho}\left(\frac{A^{2}}{\rho^{2}}-\frac{\partial p}{\partial \rho}\right)$.

The existence or nonexistence of solitary waves and stationary waves for a particular physical system can be understood through the standard methods of phase plane analysis $[7,8]$. In this method we reduce the system to a single equation of form (9). The properties of the function $\mathscr{F}(\rho)$ determine the properties of the wave solutions $\rho(\xi)$ in a fairly simple manner. Physically meaningful solutions must have $\mathscr{F} \geqslant 0$. For the systems considered here, the field $\rho$ is a mass density and must always be positive. Thus, physically relevant solutions exist when $\mathscr{F}(\rho)$ is positive over a range of positive densities. Wave solutions exist when $\mathscr{F}(\rho)$ is positive between two zeroes of the 
function $\mathscr{F}$, where the zeroes correspond to maximum and minimum densities of the wave profile. The nature of the zeroes of $\mathscr{F}$ determines the nature of the wavelike solutions. For example, if $\mathscr{F}$ is positive between two simple zeroes, then (ordinary) nonlinear waves result. However, if $\mathscr{F}$ is positive between a simple zero and a double zero (where both $\mathscr{F}$ and $\partial \mathscr{F} / \partial \rho$ vanish $)$, then solitary wave solutions can be found [7-10].

\section{Examples of charge density theories}

As we show in this section, many physical systems can be described by charge density theories. The simplest example of a nontrivial charge density is given by

$q(\rho)=\rho-\rho_{0}$,

where $\rho_{0}$ is the dimensionless background density of the fluid. We can obtain this charge density by subtracting out the contribution to the gravitational potential due to the background fluid; this charge density theory thus reproduces the original approximation of Jeans $[3,11]$. Another way to obtain a theory with the above mathematical form is to consider a physical system which rotates at a uniform rate $\Omega$ [2]. In the direction perpendicular to the rotation axis, Gauss's law for a uniform density cylinder with density $\rho_{0}$ implies

$\varpi \Omega^{2}=2 \pi G \rho_{0} \varpi$

where $\varpi$ is the radial coordinate and where all quantities have their usual dimensions. Thus, the uniform density state will be in mechanical balance provided that

$\rho_{0}=\Omega^{2} / 2 \pi G$.

For waves propagating along the axis of rotation in this system, we obtain equations of motion with a "charge density" of form (11) with $\rho_{0}$ given by eq. (13).

We can also derive a charge density for theories in which gravity is modeled by a Yukawa potential. The motivation for this approximation is to incorporate a decreasing gravitational field strength while retaining a one-dimensional treatment of the problem.
In particular, we write the Poisson equation in the generalized form

$\frac{\partial^{2} \psi}{\partial x^{2}}=m^{2} \psi+\rho$

This theory produces an exponential falloff in the gravitational force between point masses; the parameter $m$ determines the effective range of the force. The right hand side of eq. (14) defines an effective charge density $q$, although it is not written explicitly as a function of density only. However, we can integrate the stationary version of the force equation (2) to obtain

$\psi+h(\rho)+\frac{1}{2} v^{2}=E$,

where $E$ is the constant of integration. The quantity $h(\rho)$ is the enthalpy and is defined by

$h(\rho)=\int^{\rho} \frac{\mathrm{d} p}{\rho}$.

The speed $v$ can be eliminated by using the solution to the continuity equation for stationary waves, i.e., $v=A / \rho$. Using these results in eq. (14), we can read off the form of the charge density, i.e.,

$q(\rho)=\rho+m^{2}\left(E-h(\rho)-\frac{A^{2}}{2 \rho^{2}}\right)$.

The motivation for introducing the extra term in the Poisson equation (14) is to allow the gravitational force to decrease with increasing distance. As we show in ref. [12], another way to obtain this general behavior is to reduce a two-dimensional theory to an effective one-dimensional theory using rather severe approximations. In particular, we assume that the velocity in the transverse direction is small compared to that in the direction of propagation and we consider a simplified treatment of the wave profile in the transverse direction. We can thus obtain yet another charge density theory with

$q(\rho)=\rho-2 \mu^{2} \frac{\partial p}{\partial \rho}$,

where $\mu$ is a parameter which determines the steepness of the wave profile in the transverse direction. The charge density (18) is qualitatively similar to that obtained from a Yukawa potential (see eq. 
(17)) and the parameters $\mu$ and $m$ play a similar role (see ref. [12] for further discussion).

The standard problem of volume density waves in a plasma can also be written in terms of a charge density theory. The equations of motion governing the dynamics of the ions are the same as eqs. (1) and (2) with the electric potential $\phi$ replacing the gravitational potential $\psi$. The Poisson equation for the electric potential (in dimensionless form) can be written

$\frac{\partial^{2} \phi}{\partial x^{2}}=\exp (\phi)-\rho$

where $\rho$ is now the ion density [13,7]. Proceeding as we did for the Yukawa potential, we can integrate the stationary version of the force equation to find the potential and then use the solution to the continuity equation to eliminate the velocity. We thus obtain a charge density theory with

$q(\rho)=\exp \left[E-h(\rho)-A^{2} / 2 \rho^{2}\right]-\rho$,

where $E$ is the constant of integration and $h(\rho)$ is the enthalpy (the pressure is often taken to be negligible in this problem and hence $h=0$ ).

Another way to obtain a charge density theory is to simulate the effects of an embedded magnetic field. In ref. [4], we derived a model equation on this basis. We assumed that the magnetic field points in a direction perpendicular to that of the wave motion and that the neutral component of the fluid is coupled to the magnetic field through its frictional interaction with the ionized fluid component (which is well coupled to the field). We also ignored any chemical effects so that the ionized component of the fluid obeys a continuity equation. The resulting model equation can be derived from a theory with the charge density written in the form

$q(\rho)=\rho+\Gamma\left(\frac{1}{\rho}-\frac{1}{\rho_{\mathrm{F}}}\right)$,

where $\Gamma$ represents the coupling strength between the neutral and ionized components and where $\rho_{\mathrm{F}}$ determines the ion density (assumed to be constant in ref. [4]). We note that the model corresponding to the charge density (21) is idealized in two ways. First, a dissipative term has been dropped to obtain this form. Second, the ion density is not calculated self-consistently. The net result of these approximations is to make the magnetic force on the neutral component into a long-range force that balances the long-range force of gravity. This treatment is unphysical in the sense that these magnetic forces, which arise from the frictional force exerted on the neutral components by the ions, are intrinsically local. On the other hand, this approximation allows for something to cancel the long-range force of gravity, which does not fall off with distance because of the onedimensional treatment of the problem (see refs. [4,12] for further discussion).

\section{General theorems and results}

In this section we present general analytic results that are applicable for arbitrary forms of the charge density $q(\rho)$. In particular, we present four elementary theorems which greatly constrain the allowed types of wave behavior for this class of theories. Detailed proofs of these results and further discussion will be given in ref. [12].

To begin, we present a theorem which shows that for any theory with a charge density $q(\rho)$, a strong constraint must be met in order for stationary waves to exist. This constraint arises from the fact that a stationary wave must have local extrema where the pressure and velocity gradients go to zero. Therefore, in order for the wave to remain stationary, the force of gravity must also vanish at these points. This behavior can only occur if the integral of the charge density $q(\rho)$ between two extrema is zero. This argument can be put in the form of a theorem for stationary waves.

Theorem 1 . If a stationary wave solution $\rho(\xi)$ exists for the one-dimensional theory, then the integral of the charge density over one wavelength must vanish, i.e.,

$Q \equiv \int_{-\lambda / 2}^{\lambda / 2} \mathrm{~d} \xi q[\rho(\xi)]=0$,

where the wavelength of a solitary wave is taken to be infinite. This claim is limited to the case of nonsingular solutions; for solitons we also require that 
the density does not vanish at spatial infinity.

Theorem 1 greatly limits the allowed types of wave behavior in systems with self-gravity. For the simplest case of $q(\rho)=\rho$, theorem 1 implies that no stationary waves or solitary waves can exist because $\rho$ is a mass density and is positive definite (the absence of solitary waves in this context was found previously in both refs. [4] and [5] ). Another important consequence of this "no-charge theorem" is that the charge density $q(\rho)$ must vanish in the asymptotic limit $\xi \rightarrow \infty$ for a solitary wave or soliton. For solitary waves, the wavelength is infinite and the mass density $\rho(\xi)$ approaches a constant as $\xi \rightarrow \infty$.

Next, we consider the relationship between the existence of solitary waves and the absence of gravitational instability. This relationship arises because a solitary wave is, in some sense, an infinite wavelength perturbation on a uniform medium. On the other hand, the presence of gravitational instability implies that all perturbations with sufficiently long wavelengths will collapse [3]. Thus, the existence of a stationary perturbation with an infinite wavelength (a solitary wave) is inconsistent with the presence of gravitational instability. This relationship can be stated more precisely as follows.

Theorem 2. Suppose a physical system obeys a generalized model equation of motion of form (8) and this model equation has solitary wave solutions. Then the system can have a uniform density state that is gravitationally stable for arbitrarily large length scales.

We note that the converse of theorem 2 is not true. Physical systems of this type can be gravitationally stable to perturbations of all wavelengths and still not have solitary wave solutions. Thus, the requirement of gravitationally stable configurations represents a necessary condition (and not a sufficient condition) for the existence of solitary waves.

The question of whether or not solitary wave and stationary wave solutions exist is fundamental to the study of nonlinear dynamics. For the class of charge density theories considered in this paper, the question becomes: What properties of the charge density $q(\rho)$ are required for the existence of solitary waves and what properties are required for the existence of stationary waves?
We first find a necessary condition on the charge density for solitary waves. When such waves exist, the first integral $\mathscr{F}$ of the equation of motion must be positive between a double zero and a ordinary zero (see eqs. (9), (10)). We restrict our discussion to waves which avoid the singularity at the sonic point (which occurs when the term in large parentheses in eq. (9) vanishes). We also restrict our discussion to waves with finite velocity; this second restriction eliminates the point $\rho=0$ as a candidate for the double zero (notice that for $\rho \rightarrow 0$, we get $v=A / \rho \rightarrow \infty$ by the continuity condition (7)). The required double zero of $\mathscr{F}$ must thus occur at a double zero of $f(\rho)$. Furthermore, $f(\rho)$ must be a local minimum at the double zero. In order for the second (ordinary) zero of $\mathscr{F}$ to exist, the function $f(\rho)$ must also have a local maximum at some other density. By definition (eq. (10) ), the derivative of $f(\rho)$ is proportional to $q(\rho)$. Since we must avoid the singularity at the sonic point, both the required minimum and the maximum of $f(\rho)$ must occur where the charge density $q(\rho)$ vanishes. We denote the location of the minimum as $\rho_{1}$ and the maximum as $\rho_{\mathrm{M}}$. In order for the first critical point to be a minimum and for the second to be a maximum, we must have the correct signs for $\mathrm{d} q / \mathrm{d} \rho$ at the critical points (where the signs depend on whether we consider subsonic or supersonic waves). This argument can be summarized as the following necessary condition on the charge density $q(\rho)$ for the existence of solitary waves.

Theorem 3. In order for solitary wave solutions to exist for the class of theories considered in this paper, the charge density $q(\rho)$ must have (at least) two zeroes $\rho_{1}$ and $\rho_{\mathbf{M}}$. For subsonic waves, $q(\rho)$ must be negative between the two zeroes; for supersonic waves, $q(\rho)$ must be positive between the zeroes. (This result applies only to nonsingular solutions for which the sonic point $\rho_{\mathrm{C}}$ does not lie between $\rho_{1}$ and $\rho_{\mathrm{M}}$.)

Theorem 3 is potentially very powerful as a test to see if solitary wave solutions exist. For example, many possible charge density functions do not have two zeroes and thus solitary wave behavior can be easily ruled out. We note, however, that theorem 3 is not sufficient to guarantee the existence of solitary waves. We must place an additional constraint on 
the charge density to make sure that the second (ordinary) zero of the function $f(\rho)$ exists. Given that $q(\rho)$ has at least two zeroes, the function $f(\rho)$ has three possible forms. The first possibility is that $f(\rho)$ has a second ordinary zero and hence ordinary solitary waves exist. The second possibility is that the function $f$ has another minimum at some density $\rho_{3}>\rho_{\mathrm{M}}$ and hence turns up at large densities; in this case we can always choose the constant of integration in eq. (10) to make $\rho_{3}$ a double zero of $f$. We thus obtain a depression solution or void solution. For the remaining possibility, $f(\rho)$ asymptotically approaches a constant and the system fails to have solitary wave solutions. This issue of the existence of the second zero of $f(\rho)$ is discussed more fully in ref. [12].

Next, we consider the simpler case of ordinary stationary waves. The required conditions on the charge density $q(\rho)$ for the existence of these waves can be stated as follows.

Theorem 4. In order for stationary nonlinear wave solutions to exist for the class of theories considered in this paper, the charge density $q(\rho)$ must have (at least) one zero $\rho_{\mathrm{M}}$. For subsonic waves, $\mathrm{d} q / \mathrm{d} \rho>0$ at $\rho_{\mathrm{M}}$ and this zero must occur at a density larger than the sonic density $\rho_{\mathrm{C}}$ (the density of the singularity). Similarly, for supersonic waves, the system must have $\mathrm{d} q / \mathrm{d} \rho<0$ at $\rho_{\mathrm{M}}<\rho_{\mathrm{C}}$.

The conditions outlined in theorem 4 are both necessary and sufficient for the existence of stationary waves. This result is straightforward to understand. In order for such waves to exist, the function $f(\rho)$ must have a maximum and two zeroes $\rho_{1}<\rho_{2}$ such that $\rho_{\mathrm{C}}<\rho_{1}$ for subsonic waves and $\rho_{\mathrm{C}}>\rho_{2}$ for supersonic waves. The zero of $q(\rho)$ is required to provide a critical point for $f(\rho)$. The sign requirement on $\mathrm{d} q / \mathrm{d} \rho$ ensures that the critical point $\rho_{\mathrm{M}}$ is a maximum. The third condition, that the critical point $\rho_{M}$ be larger than the sonic point for subsonic waves and smaller than the sonic point for supersonic waves, is required so that the singularity does not lie in the range of densities of the wave profile.

\section{Summary and discussion}

In this Letter we have developed methods to study wave motions in self-gravitating fluids in one spatial dimension. In particular, we have introduced the concept of "charge density" for the study of such systems. In this formalism, the mass density is replaced by a "charge density" $q(\rho)$ on the right hand side of the Poisson equation for the gravitational potential (the continuity equation and the force equation remain as usual). We have shown that many physical systems can be modeled using charge density theories. In addition, we have shown that the standard example of volume density waves in a plasma can also be written as a charge density theory (where the electric potential replaces the gravitational potential). We can thus study a wide range of physical systems and physical effects using this method, which results in a simple semi-analytic theory.

We have presented a "no-charge theorem" (theorem 1) which shows that no solitary waves or stationary waves can exist in one-dimensional self-gravitating fluids unless the total charge vanishes (where the total charge is the integral of the charge density over one wavelength). This requirement of vanishing total charge greatly constrains the types of model equations that allow for stationary wave behavior.

We have shown that in order for a physical system to exhibit solitary wave behavior, the system must also be capable of a configuration which is gravitationally stable to perturbations of arbitrarily large wavelengths (see theorem 2 ). We have thus discovered a fundamental relationship between gravitational stability and the existence of solitary waves.

We have found necessary and sufficient conditions on the form of the charge density $q(\rho)$ for the existence of solitary waves and ordinary stationary waves (see theorems 3 and 4 ). These conditions allow us to determine (or at least constrain) the possible types of wave behavior for any charge density theory without having to solve the equations of motion.

In summary, we have introduced a new class of "charge density theories" for the study of wave motions in self-gravitating fluids and related systems. We have argued that many physical systems can be modeled with charge density theories. Finally, we have proven general results (theorems $1-4$ ) which provide powerful constraints on the allowed types of 
wave behavior in these systems. In a forthcoming paper [12], we present proofs of the theorems and use the results to study several examples of charge density theories.

One primary motivation of this work was to understand wave motions in self-gravitating astrophysical systems, such as star forming regions in molecular clouds $[12,14]$. However, other physical systems can be modeled with charge density theories (see, e.g., eq. (20) and the results of this paper should thus be applicable to many other problems. In addition to various direct applications of these results [12], further work should be done on general charge density theories. In particular, the related issues of wave stability [15] and wave excitation [16] should be addressed.

\section{Acknowledgement}

We would like to thank Ira Rothstein and Michael Weinstein for useful discussions. This work was supported by NASA Grant no. NAGW-2802 and by funds from the Physics Department at the University of Michigan. MF is supported by a Compton GRO Fellowship from NASA.

\section{References}

[1] G.B. Whitham, Linear and nonlinear waves (Wiley, New York, 1974).

[2] F.H. Shu, Gas dynamics (University Science Books, Mill Valley, 1992).

[3] J.H. Jeans, Astronomy and cosmogony (Cambridge Univ. Press, Cambridge, 1928).

[4] F.C. Adams and M. Fatuzzo, Astrophys. J. 403 (1993) 142.

[5] E.P.T. Liang, Astrophys. J. 230 (1979) 325.

[6] G. Götz, Class. Quantum Grav. 5 (1988) 743.

[7] E. Infeld and G. Rowlands, Nonlinear waves, solitons, and chaos (Cambridge Univ. Press, Cambridge, 1990).

[8] P.G. Drazin and R. S. Johnson, Solitons: an introduction (Cambridge Univ. Press, Cambridge, 1989).

[9] S. Coleman, Aspects of symmetry (Cambridge Univ. Press, Cambridge, 1985).

[10] R. Rajaraman, Solitons and instantons: an introduction to solitons and instantons in quantum field theory (NorthHolland, Amsterdam, 1987).

[11] J. Binney and S. Tremaine, Galactic dynamics (Princeton Univ. Press, Princeton, 1987).

[12] F.C. Adams, M. Fatuzzo and R. Watkins, submitted to Astrophys. J. (1993).

[13] R. C. Davidson, Methods in nonlinear plasma theory (Academic Press, New York, 1972).

[14] F.H. Shu, F.C. Adams and S. Lizano, Annu. Rev. Astron. Astrophys. 25 (1987) 23.

[15] R.L. Pego and M.I. Weinstein, Phys. Lett. A 162 (1992) 263.

[16] J. Arons and C. Max, Astrophys. J. Lett. 196 (1975) L77. 